\title{
Oral HPV infection in a clinic-based sample of Hispanic men
}

Vivian Colon-López ${ }^{1,2^{*}}$, Valerie Quiñones-Avila², Lizbeth M Del Toro-Mejías ${ }^{3}$, Keysha Reyes ${ }^{4}$, Manuel E Rivera ${ }^{5}$, Kathleen Nieves ${ }^{5}$, María M Sánchez-Vazquez ${ }^{6}$, Magaly Martínez-Ferrer ${ }^{6,7}$ and Ana P Ortiz ${ }^{1,8}$

\begin{abstract}
Background: Human papillomavirus (HPV) is associated to the pathogenesis of various cancers, such as oropharyngeal squamous cell carcinoma, which has a high incidence in Puerto Rican men. Despite the burden of oral cancer in Puerto Rico, little is known about the epidemiology of oral HPV infection, particularly in high-risk men. Therefore, this study is aimed at determining the prevalence of oral HPV infection, the genotype distribution and correlates associated with oral HPV infection in men of at least 16 years of age attending a sexually transmitted infection (STI) clinic in Puerto Rico.
\end{abstract}

Methods: A cross-sectional study consisting of 205 men was conducted. Participants provided a 30-second oral rinse and gargle with mouthwash. Following DNA extraction, HPV genotyping was performed in all samples using Innogenetics Line Price Assay (INNO-LiPA). A questionnaire was administered, which included a demographic, behavioral and a clinical assessment. Descriptive statistics and bivariate analysis were used to characterize the study sample. Variables that achieved statistical significance in the bivariate analysis $(p<0.05)$ were assessed in multivariate logistic regression models.

Results: The mean age of the study sample was $38.5 \pm 14.2$ years. Oral HPV prevalence among men was $20.0 \%$ $(95.0 \% \mathrm{Cl}=14.8 \%-26.1 \%)$ and of HPV type 16 was $2.4 \%(95.0 \% \mathrm{Cl}=0.8 \%-5.6 \%)$. Oral HPV prevalence significantly increased over increasing age categories ( $p$-trend $=0.001$ ). Multivariate analysis showed that oral HPV was independently associated with number of sexual partners (adjusted $\mathrm{OR}=1.02 ; 95 \% \mathrm{Cl}=1.01-1.03$ ) and lifetime use of cigarettes (adjusted $\mathrm{OR}=3.00 ; 95 \% \mathrm{Cl}=0.98-9.16$ ).

Conclusions: Oral HPV among the sampled men in the STI clinic was high, regardless of the HIV status or sexual behavior. Interventions in STI clinics should include screening for HPV in the oral cavity for the early detection and reduction of long-term consequences of oral HPV infection, such as oropharyngeal cancer.

Keywords: Oral HPV infection, Epidemiology, High-risk men, STI/STD clinic, Puerto Rico

\section{Background}

Human papillomavirus (HPV) infection is considered the most common sexually transmitted infection (STI) [1]. About 6 million people are diagnosed each year and approximately $9.0-13.0 \%$ of the world population $(630$ million people) is already infected with the disease [2]. HPV plays a role in the pathogenesis of Head and Neck Squamous Cell Carcinomas (HNSCCs) and Oropharyngeal Squamous Cell Carcinomas (OPSCCs) $[3,4]$. The

\footnotetext{
*Correspondence: vivian.colon@upr.edu

${ }^{1}$ Cancer Control and Population Sciences Program, University of Puerto Rico Comprehensive Cancer Center, San Juan, Puerto Rico

${ }^{2}$ Department of Health Services Administration, Graduate School of Public Health, University of Puerto Rico, San Juan, Puerto Rico

Full list of author information is available at the end of the article
}

incidence and mortality rates of HNSCCs have increased in several countries over the last three decades [5-9]. These group of cancers are clinically characterized by the presence of oncogenic high-risk (HR) HPV, predominantly type 16 , that has been detected approximately in $95.0 \%$ of positive tumors $[10,11]$. In head and neck cancers, studies have shown that the prevalence of HPV DNA varies by cancer site and geography [12]. HPV DNA has been found in $35-50 \%$ in developed countries in contrast with the rest of the oral cavity, where HPV DNA is found in $5-15 \%$ of the cases [12]. HNSCCs have broad varying rates of incidence and mortality worldwide, with higher rates in European countries and Southeast Asia [13]. According to data from nine Surveillance, 
Epidemiology, and End Results (SEER) program registries from 1973 to 2004, in the United States (US), the incidence of HNSCC increased particularly in young and middle aged individuals ( $<60$ years) and men [14]. This cancer type is currently the eighth most common cancer among men in the US $[15,16]$.

Some studies have found several risk factors that increase the risk of HPV in the oral cavity. Tobacco smoking and alcohol consumption are the main risk factors for HNSCC $[15,16]$. Although the incidence of HPV-unrelated OPSCC associated with tobacco and alcohol use has declined over recent decades, OPSCC related to HPV has significantly increased [4]. These increases in the prevalence of HPV in the oral cavity may be the result of changes in sexual behaviors [10]. Oral HPV prevalence is more than 8-fold higher among sexually experienced individuals as compared to sexually inexperienced individuals and increases significantly with number of sexual partners [17]. This prevalence has been as high as $20.0 \%$ among individuals who have reported more than 20 lifetime sexual partners during their lifetime [17]. Additionally, men with multiple lifetime male sex partners (MSM) are 2 to 4 times more likely to be HPV 6 or 11, seropositive and 3 to 11 times more likely to be seropositive to HPV 16 or 18 [18]. Also, high rates of oral HPV infection have been found in HIV (Human immunodeficiency virus) seropositive individuals $[1,10,19,20]$. A study has also shown the HPV prevalence to be higher among current smokers (20.7\%) and heavy alcohol drinkers (12.3\%) [17].

Studies concerning the epidemiology of oral HPV infection in men are limited since much of the research focuses on factors associated with cervical cancer in women $[21,22]$. However, as the causes for the excess risk of HPV-related cancers in the US remains unclear [17], it is important to determine epidemiology the for this group. For example, although a substantial percentage of oropharyngeal cancer is attributed to alcohol (7.0-19.0\%) and tobacco exposure (25.0\%) [23], in the US about one quarter of the oral cancer cases in men and approximately half of those in women in Puerto Rico are not attributed to these exposures [24]. These findings for Puerto Rico are consistent with US data [25] and highlight the need to further understand the epidemiology of HPV infection in the oral cavity, as well as its relationship with oral cancers and anogenital infection.

Furthermore, the limited epidemiological and behavioral research on oropharyngeal HPV infection in men, including the high-risk groups, may delay the development of effective interventions to reduce the burden of oral HPV infection. For Puerto Rico in particular, men have had a higher incidence and mortality of oropharyngeal cancer as compared with Hispanics in the US [26]. According to the Puerto Rico Central Cancer Registry, from 2005-2009, this cancer type was considered the fourth most common cancer among men in Puerto Rico [27]. Puerto Rican men also have had a higher mortality risk of the disease as compared with non-Hispanic whites and non-Hispanic blacks in the US [26].

Therefore, given the burden of oropharyngeal cancer in Puerto Rico, the aim of this study was to (1) determinate the prevalence of HPV infection in the oral region and (2) to identify socio-demographic, behavioral and clinical risk factors associated with oral HPV infection in men attending a STI clinic in San Juan, Puerto Rico. STI clinics can be an ideal venue for targeting oral HPV infection epidemiology in high risk groups of men, such as men who have sex with men (MSM), HIVpositive men, and men with STIs other than genital warts. Therefore, data collected from this venue may offer the opportunity to reach these vulnerable and underserved populations more efficiently than generalpopulation studies.

\section{Methods}

\section{Study design and research procedures}

This study consisted of a cross-sectional study with men attending a publicly funded STI clinic in San Juan, Puerto Rico. A convenience sample was drawn from 205 men from within the clinic's waiting room, coupled with subsequent screening to confirm eligibility (at least 16 years of age) and informed consent. Study participation was voluntary and written informed consent, including Health Insurance Portability and Accountability Act (HIPAA) forms, notifying about the nature of the study, general purpose, the types of research and clinical procedures involved, were provided. All study design, written consent and data collection procedures were approved by the University of Puerto Rico-Medical Sciences Campus (UPR MSC) Institutional Review Board (IRB).

\section{Behavioral interview}

The behavioral interview was completed in approximately 25 minutes by a trained interviewer. Domains included demographic characteristics (e.g. age, place of birth, current residence, employment, and educational attainment), detailed assessment of sexual risk (e.g. including age of onset, current sexual partners and practices), and assessment of alcohol, cigarette and drug use- (e.g. types, frequency, and mode of administration of illicit/illegal drugs such as: marihuana, cocaine, heroin, and crack). Additionally, the assessment included questions about health history (self-reported STI's and HIV status).

\section{Variables of interest}

A person was considered oral HPV positive if resulted positive to any of the 27 types tested, whereas all others were considered negative. Behavioral characteristics included patterns of smoking tobacco, smoking marihuana, 
and drinking. Lifetime and recent cigarette and marihuana smoking, as well as lifetime alcohol use were measured. Lifetime smoking was defined as having smoked at least once during the lifetime. Current smoking was defined as smoking at least one cigarette per day for the past 12 months. Years smoking was defined as the number of years spent smoking regularly. Lifetime smoking of marihuana was measured as "ever smoked marihuana", which referred to having smoked marihuana at least once during lifetime. "Ever smoked marihuana in the past 12 months" was defined as having smoked marihuana at least once during the past 12 months. Lifetime alcohol use was measured as "ever having an alcoholic beverage", which referred to drinking at least once during lifetime.

The self-reported sexual characteristics analyzed within the sample dealt with sexual behavior, including lifetime and recent oral sex. Men who had sex with men (MSM) was a self-reported measure from the question: "Regarding your lifetime sexual partners, how many of them have been men?" Those participants that answered " 1 or more men", were categorized as "men who had sex with men" (MSM) in their lifetime. Those that answered zero (0) were categorized as non-MSM. Lifetime oral sex was defined as having had oral sex at least once during lifetime. Recent oral sex was defined as having had oral sex at least once during the past 12 months.

\section{Oral HPV specimen collection}

All of the specimen collection and HPV polymerase chain reaction (PCR) procedures used in this study have been extensively tested and refined, and used in multiple previously published studies [28,29]. After completion of the behavioral interview, participants underwent collection of biological specimens by trained physicians at the study site. An oral mouthwash sample was collected to obtain DNA for HPV genotyping. Oral rinse samples were collected as previously described in the National Health and Nutrition Examination Survey (NHANES) [30]. Briefly, each participant vigorously swished and gargled $10 \mathrm{ml}$ of Scope mouthwash for 30 seconds. This was followed by depositing the Scope and saliva generated from the mouthwash into a specimen collection cup.

\section{DNA extraction/ inno-LiPA HPV genotyping testing}

DNA was extracted using the DNA purification from buccal cells protocol from Gentra PureGene Kit (Qiagen, Valencia, CA) and following the manufacturer's instructions. The concentration of each DNA was quantified and qualified spectrophotometrically using a Nanodrop HPV genotyping was determined in all study samples using the INNO-LiPA HPV Genotyping Extra assay (Innogenetics, Belgium) and following the manufacturer's instructions. The INNO-LiPA HPV assay is a PCR-based hybridization assay that includes a cocktail of biotinylated consensus primers (small primer fragment (SPF) 10) to amplify a portion of the L1 open reading frame (ORF) of 13 established HR HPV types $(16,18,31,33,35,39,45,51,52,56,58,59,66), 5$ known or putative HR types $(26,53,68,73$, and 82$), 7$ LR HPV types $(6,11,40,43,44,54$, and 70), additional non-differentiated HPV types, and types with undefined risk (74 and 69/71). After DNA extraction, all specimens were subjected to PCR amplification (40 cycles) using the Inno-LiPA HPV Genotyping Extra Amp. The PCR product was then denatured, and a $10-\mu \mathrm{l}$ aliquot was hybridized onto nitrocellulose strips where the HPV typespecific oligonucleotides were already bound. After $60 \mathrm{~min}$ at $49^{\circ} \mathrm{C}$, the PCR product bound to a specific probe was detected by an alkaline phosphatase-streptavidin conjugate and colorimetric detection. Results were visually interpreted by experienced laboratory personnel using the reference guide provided. Some types are defined by a single positive probe on the genotyping strip (i.e., HPV6, -11, and -16), but others are interpreted as a combination of two to four probes (i.e., HPV18, -33, and -58). While the manufacturer provides interpretation of type detection, including "possible" types, only those that were detected unequivocally were included in the analysis.

\section{Statistical analysis}

Frequency distributions and descriptive statistics were first used to characterize the study sample in terms of demographics, behavioral and HIV status. Overall prevalence of oral HPV infection was estimated, along with 95.0\% binomial confidence interval (CI). Frequency distributions were used to describe the DNA HPV-types detected in the oral cavity. Chi-square tests, Fisher's exact tests, and independent t-tests were used to evaluate differences in demographic, behavioral, and HIV status. Statistical significance was set at p-value $<0.05$. Variables that resulted significantly associated with oral HPV seroprevalence in the bivariate analyses were later evaluated for possible collinearity. A Pearson correlation of 0.5 or greater and a p-value for the two-tailed significance test $<0.05$ was considered as a significant correlation.

Factors significantly associated with oral HPV infection were included in a logistic regression analysis. Different binary logistic regression models were developed in order to investigate the relationship between the selected risk factors and the occurrence of any type of oral HPV infection. Associations with oral HPV prevalence were reported as crude and adjusted Odds Ratios (ORs), along with $95 \%$ CIs and significance assessed through the Wald test $\mathrm{p}$-value $<0.05$. Trend tests were conducted across age categories. Goodness of fit for all models was assessed using the log likelihood estimates. In order to choose the best model, the log likelihood estimates were 
compared by subtracting each model's log likelihood from the base model's log likelihood. First-order interactions between the significant factors of the chosen model were also evaluated in the logistic regression analysis.

Statistical analyses were performed using the statistical package SPSS (Version 21.0, Chicago, IL), while 95\% confidence intervals $(\mathrm{CI})$ for prevalence estimations were performed using the Binomial CI Calculator from STATA Statistical Software (Release 11, College Station, TX).

\section{Results}

\section{Participant characteristics}

The demographic and behavioral characteristics of the 205 participating men are shown in Table 1 . The mean age of the study sample was 38.5 years (standard deviation $[S D]=14.2)$. The majority $(65.2 \%)$ had a high school education or less and was employed (53.9\%). Also, public health insurance was predominant among the sample (58.9\%); although $18.3 \%$ reported not having any type of medical insurance.

The mean years for smoking was 23.9 years $(\mathrm{SD}=14.3)$. More than half of the interviewed men had smoked at least once in their lifetime (73.5\%), reported to be current smokers (65.8\%), and had at least one alcoholic beverage in their lifetime (95.1\%). Though the majority had smoked marihuana at least once in their lifetime (62.3\%), they reported not having smoked marihuana in the past 12 months (55.2\%).

Half of the sample reported having HIV (50.2\%). The mean years of living with HIV was 11.5 years $(\mathrm{SD}=$ 8.4 years). Oral HPV infection was observed among $22.3 \%$ of the HIV positive men and $17.8 \%$ among HIV negative men. Additionally, approximately a third (29.5\%) of the participants indicated having had sex with another man (MSM). The vast majority of the MSM's interviewed in this sample had reported oral sex at least once in their lifetime (91.2\%) and at least once in the past 12 months (91.7\%). These results were also consistent with those who reported being heterosexual, in which a vast majority (91.9\%) reported having had oral sex at least once in their lifetime and $76.0 \%$ having had oral sex at least once in the past 12 months.

\section{Oral HPV prevalence}

The prevalence estimates for oral HPV infection by the 27 individual genotypes analyzed among the 205 participating men aged 16-81 years are shown in Table 2. The prevalence of oral HPV infection among men was 20.0\% (95\% CI = 14.8\%, 26.1\%). The prevalence of high-risk HPV infection was $10.7 \%(95 \% \mathrm{CI}=6.8 \%, 15.8 \%)$ and for low-risk HPV was $7.3 \%$ (95\% CI $=4.2 \%, 11.8 \%$ ). The most prevalent high-risk subtype was HPV-52 (2.9\%), while the most prevalent lowrisk subtype was HPV-6 (3.9\%). HPV types 18, 26, 33, 45, $53,59,79 / 71$, and 82 were not detected in this sample.

\section{Factors associated with oral HPV infection in bivariate analysis}

Table 3 shows the results for the associations between demographic, behavioral, and clinical characteristics with HPV status. Oral HPV prevalence was significantly associated with age, lifetime smoking, years smoking, lifetime marihuana use, and lifetime number of sexual partners. However, bivariate correlation analyses demonstrated that having smoked at least once during lifetime, having smoked marihuana at least once during lifetime, and years smoking were significantly correlated (p-value <0.05). Upon this result, we only included "having smoked at least once during lifetime" for subsequent analyses.

\section{Factors associated with oral HPV infection in logistic regression analyses}

Results for the univariate and multivariate logistic regression models evaluating the association of demographic and behavioral variables with the likelihood of HPV infection are demonstrated in Table 4. In the unadjusted logistic regression model, factors independently associated with increased prevalence odds of HPV infection included: age, lifetime smoking and lifetime number of sexual partners. The prevalence of any type of oral HPV significantly increased over increasing age categories $(\mathrm{p}$-trend $=0.001)$. Men who had smoked at least once in their lifetime were three times more likely to test positive for any HPV type infection $(\mathrm{OR}=3.10$; $95 \% \mathrm{CI}=$ $1.15,8.36)$ as compared with those who had never smoked in their lifetime. Also, per every unit increase in lifetime sexual partners, the prevalence of oral HPV infection increased by $2 \%$.

In the complete multivariate model (model 3), lifetime smoking and lifetime sex partners remained independently associated with detection of any type of oral HPV. Odds of detecting any type of oral HPV infection remained three-fold higher among men who had smoked at least once in their lifetime compared to those who had never smoked $(\mathrm{OR}=3.00 ; 95 \% \mathrm{CI}=0.98,9.16)$, after adjusting for differences in age and number of lifetime sexual partners. This association was marginally significant. Odds of detecting any type of oral HPV infection remained significantly higher among men with increased number of lifetime sex partners $(\mathrm{OR}=1.02$; $95 \% \mathrm{CI}=1.01,1.03)$, after adjusting for differences in age and lifetime smoking. No significant first-order interactions were observed between age, lifetime smoking, and lifetime sex partners.

\section{Discussion}

The prevalence of any type of oral HPV infection among men participating in this study was $20.0 \%(95 \% \mathrm{CI}=$ 14.8\%-26.1\%). After multivariate regression analysis, any 
Table 1 Demographic and risk related characteristics among 205 HIV+/HIV- men attending an STI clinic in Puerto Rico

\begin{tabular}{lll}
\hline Characteristic & $\begin{array}{l}\text { Number of } \\
\text { subjects }\end{array}$ & $\begin{array}{l}\text { Frequency } \\
\text { (\%) }\end{array}$ \\
\hline Demographics
\end{tabular}

Demographics

Age at recruitment

Mean (SD) 38.5 (14.2)

$16-24$
$25-34$
$35-44$
$45-54$
$55+$

Level of education

$<$ High School

High School

$>$ High School

Employed

$$
\text { No }
$$$$
\text { Yes }
$$

Type of health insurance

$\begin{array}{lll}\text { None } & 37 & 18.3 \\ \text { Public } & 119 & 58.9 \\ \text { Private } & 46 & 22.8\end{array}$

Behavioral

Lifetime smoking

$\begin{array}{lll}\text { No } & 54 & 26.5 \\ \text { Yes } & 150 & 73.5\end{array}$

Current smoker

No

Yes

$\begin{array}{ll}41 & 34.2 \\ 79 & 65.8\end{array}$

Years smoking

Mean (SD) 23.9 (14.3)

Ever had an alcoholic beverage

$\begin{array}{lll}\text { No } & 10 & 4.9 \\ \text { Yes } & 194 & 95.1\end{array}$

Ever smoked marihuana

$\begin{array}{lll}\text { No } & 77 & 37.7 \\ \text { Yes } & 127 & 62.3\end{array}$

Ever smoked marihuana in the past 12 months

$$
\begin{array}{ll}
\text { No } & 69 \\
\text { Yes } & 56
\end{array}
$$

Sexual activity

MSM

$\begin{array}{lll}\text { No } & 136 & 70.5 \\ \text { Yes } & 57 & 29.5\end{array}$

Table 1 Demographic and risk related characteristics among 205 HIV+/HIV- men attending an STI clinic in Puerto Rico (Continued)

MSM who had oral sex at least once in their lifetime

$\begin{array}{lll}\text { No } & 5 & 8.8 \\ \text { Yes } & 52 & 91.2\end{array}$

MSM who had oral sex at least once in the past 12 months

$\begin{array}{rll}\text { No } & 3 & 8.3 \\ \text { Yes } & 33 & 91.7 \\ \text { MSW } & & \\ \text { No } & 30 & 15.8 \\ \text { Yes } & 160 & 84.2\end{array}$

MSW who had oral sex at least once in their lifetime

$\begin{array}{lll}\text { No } & 13 & 8.1 \\ \text { Yes } & 147 & 91.9\end{array}$

MSW who had oral sex at least once in the past 12 months

$\begin{array}{lll}\text { No } & 29 & 24.0 \\ \text { Yes } & 92 & 76.0\end{array}$

Clinical

HIV Status

$\begin{array}{lll}\text { No } & 101 & 49.3 \\ \text { Yes } & 103 & 50.2\end{array}$

Years living with HIV

Mean (SD) 11.5 (8.4)

Any type of HPV

$\begin{array}{lll}\text { No } & 164 & 80.0 \\ \text { Yes } & 41 & 20.0\end{array}$

High risk HPV

$\begin{array}{lll}\text { No } & 183 & 89.3 \\ \text { Yes } & 22 & 10.7\end{array}$

Only high risk HPV

$\begin{array}{lcl}\text { No } & 188 & 91.7 \\ \text { Yes } & 17 & 8.3\end{array}$

Low risk HPV

$\begin{array}{lll}\text { No } & 190 & 92.7\end{array}$

$\begin{array}{lll}\text { Yes } & 15 & 7.3\end{array}$

Only low risk HPV

$\begin{array}{lll}\text { No } & 195 & 95.1\end{array}$

$\begin{array}{lll}\text { Yes } & 10 & 4.9\end{array}$

Multiple HPV

No $\quad 194 \quad 94.6$

\begin{tabular}{lll} 
Yes & 11 & 5.4 \\
\hline
\end{tabular}

type of oral HPV prevalence was significantly higher among men with a higher mean number of lifetime sexual partners. A series of studies on oral HPV prevalence 


\begin{tabular}{|c|c|c|}
\hline HPV type & $\mathrm{n}(\mathrm{N}=205)$ & $\%(95 \% \mathrm{Cl})$ \\
\hline Any HPV type & 41 & $20(14.8-26.1)$ \\
\hline High-Risk (HR) & 22 & $10.7(6.8-15.8)$ \\
\hline 16 & 5 & 2.4 \\
\hline 31 & 1 & 0.5 \\
\hline 35 & 3 & 1.5 \\
\hline 39 & 3 & 1.5 \\
\hline 51 & 2 & 1.0 \\
\hline 52 & 6 & 2.9 \\
\hline 56 & 3 & 1.5 \\
\hline 58 & 1 & 0.5 \\
\hline 66 & 2 & 1.0 \\
\hline 68 & 2 & 1.0 \\
\hline 73 & 1 & 0.5 \\
\hline 74 & 4 & 2.0 \\
\hline Low-Risk (LR) & 15 & $7.3(4.2-11.8)$ \\
\hline 6 & 8 & 3.9 \\
\hline 11 & 1 & 0.5 \\
\hline 43 & 1 & 0.5 \\
\hline 44 & 4 & 2.0 \\
\hline 54 & 1 & 0.5 \\
\hline 70 & 1 & 0.5 \\
\hline Multiple† & 11 & $5.4(2.7-9.4)$ \\
\hline
\end{tabular}

tThose that have two or more HPV types.

[31-35] have recently sampled specific populations (e.g. MSM or HIV + men) from which results of oral HPV prevalence have varied. For example, in Melbourne, Australia the prevalence of oral HPV among MSM's attending a sexual health center was $13.0 \%$ [35]. This prevalence resulted slightly lower than that reported in similar study settings among MSM's in Amsterdam (24.4\%) [34] and among HIV-infected MSM's in Italy (21.3\%) [36]. Although comparisons with other studies are not possible due to differences in the sample, the prevalence of oral HPV among MSM in our study was $19.3 \%$.

In our study, although a higher prevalence of oral HPV infection was observed among HIV positive men (22.3\% in HIV-positive vs. $17.8 \%$ in HIV-negative), this association was not significant. Also, neither sexual behavior nor oral sexual practices were significantly associated with any type of oral HPV detection. This may be explained by the selection of a high sexual risk group of men attending a public STI clinic, which lacked enough heterogeneity with regards to some risk factors.

Prevalence of oral HPV significantly increased within increasing age categories. The peak prevalence was found among men aged $55-81$ years of age (34.5\%). This increasing trend could be explained by an increased oral HPV incidence with age [17], persistent and/or reactivated oral HPV infections [31] due to loss of immunity as age increases [17,37], and/or increased persistence among older individuals $[17,38]$. However, it is important to clarify that although a trend was found between oral HPV and age, the association between these variables did not remain statistically significant after adjusting for lifetime sex partners and lifetime smoking in the multivariate logistic regression model.

The multivariate logistic regression model for any type of oral HPV infection demonstrated an independent significant association with lifetime number of sex partners and a marginal independent association with lifetime smoking, after adjusting for differences in age categories. Increased odds of any type of oral HPV infection among men with increased number of sex partners is supported by the literature [17], indicating that oral HPV infection is higher among sexually experienced individuals than by nonsexual contact. The association with lifetime smoking, though marginal, may confirm the biological plausibility associated with oral HPV infection [33]. Cigarette smoking inhibits a variety of immune responses in the oral cavity [39], which may allow for increased HPV persistence [33]. The oral epithelial abrasion due to the direct exposure to the carcinogens in tobacco may also represent the pathway for increased HPV detection among smokers [35].

The findings of this study need to be interpreted with caution because of its inherent limitations. We acknowledge that perhaps the sample size of 205 men was not sufficient for this type of study. Though this number can be considered fair in other types of research, we found it to be insufficient for this type of setting, especially when creating categories for certain outcome variables and in analyses for associations with clinical characteristics.

Oral HPV infection was measured once, so it was not possible to differentiate between newly acquired, persistent, or reactivated infections [31]. Site of infection, such as the oral cavity or the oropharynx, was also not possible to differentiate, due to the oral rinse sampling technique used [31]. We recognize additional limitations in the way some of the behavioral and clinical variables were measured. This study relied upon self-reported data for HIV status, which could lead to misclassification and residual confounding. Among HIV positive men, CD4 T-cell count, viral load and highly active antiretroviral therapy (HAART) use, was not available. Also, the lack of frequency measures for alcohol, tobacco, and marihuana use limited the interpretation of the results. Perhaps measuring recent use, within the past 6 or 12 months, and measuring the quantity of use for these behavioral variables could have provided a more detailed 
Table 3 Oral HPV prevalence and risk factors among 205 HIV+/HIV- men attending an STI clinic in Puerto Rico

\begin{tabular}{llll}
\hline Characteristic & HPV negative & HPV positive & p-value \\
& $\%(n)$ & $\%(n)$ \\
\hline
\end{tabular}

Demographic

Age at recruitment

$\begin{array}{lll}16-24 & 88.6(39) & 11.4(5) \\ 25-34 & 97.6(40) & 2.4(1) \\ 35-44 & 73.2(30) & 26.8(11) \\ 45-54 & 71.7(33) & 28.3(13) \\ 55+ & 65.5(19) & 34.5(10)\end{array}$

Level of education

$<$ High School
High School
$>$ High School
Employed

$\begin{array}{lll}\text { No } & 79.1(34) & 20.9(9) \\ \text { Yes } & 82.7(91) & 17.3(19)\end{array}$

Type of Health Insurance

$\begin{array}{lll}\text { None } & 83.8(31) & 16.2(6) \\ \text { Public } & 78.2(93) & 21.8(26) \\ \text { Private } & 80.4(37) & 19.6(9)\end{array}$

Behavioral

Ever smoked

$\begin{array}{lll}\text { No } & 90.7(49) & 9.3(5) \\ \text { Yes } & 76.0(114) & 24.0(36)\end{array}$

0.020

Current smoker

$\begin{array}{clll}\text { No } & 73.2(30) & 26.8(11) & 0.514 \\ \text { Yes } & 78.5(62) & 21.5(17) & \\ \text { Years Smoking } & 75.0(87) & 25.0(29) & \\ \text { Mean (SD) } & 22.3(14.5) & 28.7(12.5) & 0.038\end{array}$

Ever had an alcoholic beverage

$\begin{array}{llll}\text { No } & 90.0(9) & 10.0(1) & 0.367 \\ \text { Yes } & 79.4(154) & 20.6(40) & \end{array}$

Ever smoked marihuana

$\begin{array}{llll}\text { No } & 87.0(67) & 13.0(10) & 0.048 \\ \text { Yes } & 75.6(96) & 24.4(31) & \end{array}$

Ever smoked marihuana in the past 12 months

$\begin{array}{lccc}\text { No } & 69.6(48) & 30.4(21) & 0.105 \\ \text { Yes } & 82.1(46) & 17.9(10) & \\ \begin{array}{l}\text { Sexual Activity } \\ \begin{array}{l}\text { Lifetime number of sexual } \\ \text { partners }\end{array}\end{array} & 79.4(158) & 20.6(41) & 0.005 \\ \begin{array}{l}\text { Mean (SD) } \\ \text { MSM }\end{array} & 20.2(28.9) & 65.9(98.2) & \\ \text { No } & & & \\ & 80.1(109) & 19.9(27) & 0.930\end{array}$

Table 3 Oral HPV prevalence and risk factors among 205 HIV+/HIV- men attending an STI clinic in Puerto Rico (Continued)

\begin{tabular}{lccc}
\hline Yes & $80.7(46)$ & $19.3(11)$ & \\
MSM who had oral sex at least once in their lifetime & \\
No & $80.0(4)$ & $20.0(1)$ & 0.673 \\
Yes & $80.8(42)$ & $19.2(10)$ &
\end{tabular}

MSM who had oral sex at least once in the past 12 months

$\begin{array}{cccc}\text { No } & 33.3(1) & 66.7(2) & 0.066 \\ \text { Yes } & 87.9(29) & 12.1(4) & \\ \text { MSW } & & & \\ \text { No } & 86.7(26) & 13.3(4) & 0.525 \\ \text { Yes } & 81.9(131) & 18.1(29) & \end{array}$

MSW who had oral sex at least once in their lifetime

$\begin{array}{llll}\text { No } & 92.3(12) & 7.7(1) & 0.276 \\ \text { Yes } & 81.0(119) & 19.0(28) & \end{array}$

MSW who had oral sex at least once in the past 12 months

$\begin{array}{llll}\text { No } & 79.3(23) & 20.7(6) & 0.688 \\ \text { Yes } & 82.6(76) & 17.4(16) & \end{array}$

Clinical

HIV

\begin{tabular}{llll} 
No & $82.2(83)$ & $17.8(18)$ & 0.422 \\
Yes & $77.7(80)$ & $22.3(23)$ & \\
Years living with HIV & $79.2(80)$ & $29.8(21)$ & 0.644 \\
Mean (SD) & $11.3(8.8)$ & $12.2(6.9)$ & \\
\hline
\end{tabular}

insight on the magnitude of association between these variables and oral HPV in men. Categorizing these variables into more detailed recent use measurements could have increased validity and power.

Also, due to the small sample size among MSM's, opportunities to determine recent MSM behavior (i.e. last 3 months) and conduct multivariable analyses were not possible. Other limitations include the lack of data regarding the use of condoms and other variables which could help us understand sexual partnering and preventive practices among MSM. Furthermore, we were unable to examine MSM sociocultural contexts, like violence, stigma, homophobia, social networks, and the construction of gender identities. Future studies need to explore these social determinants when attempting to understand the sexual behavior and HPV/HIV-related risks to which the participants of this study setting are exposed in Puerto Rico.

Since the study was conducted in a high risk STI public clinic, our findings are unlikely to be generalizable to populations at lower sexual risk. However, data collected from a highly selective population, such as a public STI clinic, may also offer an opportunity to reach targeted high-risk groups more efficiently than general population-based 
Table 4 Logistic regression results for associations with HPV among 205 men attending an STI clinic in Puerto Rico

\begin{tabular}{|c|c|c|c|c|c|c|c|c|}
\hline Variable & Unadjusted OR (95\% CI) & $p$-value & Model 1 Adjusted OR (95\% Cl) & $p$-value & Model 2 Adjusted OR (95\% Cl) & p-value & Model 3 Adjusted OR (95\% Cl) & $\mathrm{p}$-value \\
\hline \multicolumn{9}{|l|}{ Age at recruitment } \\
\hline $16-24$ & 1.00 & & 1.00 & $0.021^{*}$ & 1.00 & $0.059^{*}$ & 1.00 & $0.074^{*}$ \\
\hline 25-34 & $0.20(0.02,1.75)$ & 0.144 & $0.15(0.02,1.40)$ & 0.097 & $0.18(0.02,1.62)$ & 0.126 & $0.13(0.01,1.22)$ & 0.075 \\
\hline $35-44$ & $2.87(0.90,9.12)$ & 0.076 & $2.28(0.70,7.43)$ & 0.172 & $2.39(0.70,8.18)$ & 0.164 & $1.86(0.54,6.46)$ & 0.328 \\
\hline $45-54$ & $3.07(0.99,9.52)$ & 0.052 & $2.68(0.85,8.47)$ & 0.093 & $2.77(8.53,8.96)$ & 0.090 & $2.28(0.69,7.51)$ & 0.174 \\
\hline $55+$ & $4.11(1.23,13.70)$ & 0.022 & $3.68(1.08,12.57)$ & 0.037 & $2.54(0.70,9.26)$ & 0.157 & $2.15(0.58,7.98)$ & 0.254 \\
\hline$P$ trend & 0.001 & & & & & & & \\
\hline Log Likelihood & 180.39 & & & & & & & \\
\hline Lifetime sex partners & $1.02(1.01,1.03)$ & $<0.001$ & & & $1.02(1.01,1.03)$ & 0.002 & $1.02(1.01,1.03)$ & 0.002 \\
\hline Log Likelihood & 180.47 & & & & 161.22 & & & \\
\hline \multicolumn{9}{|l|}{ Lifetime smoking } \\
\hline No & 1.00 & & 1.00 & & & & 1.00 & \\
\hline Yes & $3.10(1.15,8.36)$ & 0.026 & $3.20(1.14,8.95)$ & 0.027 & & & $3.00(0.98,9.16)$ & 0.054 \\
\hline Log Likelihood & 198.64 & & 174.56 & & & & 156.87 & \\
\hline
\end{tabular}

overall p-value (Wald). 
studies. As described in other studies [40,41], participants attending publicly funded STI clinics are likely to be young, uninsured and poor. Therefore, this scenario has helped target oral HPV infection analyses among a vulnerable population.

To our knowledge, this is the first study currently examining the epidemiology of oral HPV infection among men attending a public STI clinic in Puerto Rico. Since previous studies have reported that men in Puerto Rico have a higher incidence and mortality of oropharyngeal cancer as compared to Hispanics in the US [26], future studies should further elucidate if potential interactions between alcohol use, tobacco use, and HPV infection may explain the higher burden of oral cancer in this population. Also, preventive efforts in this area are warranted. However, primary preventive interventions in this high sexual risk setting can be too late and ineffective. Since our study demonstrated a high oral HPV prevalence, capacity building and training on malignancies in the oral cavity among the medical staff are recommended. This type of intervention in a high risk setting can be appropriate to reduce complications and comorbidities.

\section{Conclusions}

This study reports on the prevalence of and risk factors associated with oral HPV infection in a sample of men attending a public STI clinic in Puerto Rico. Oral HPV prevalence was high, regardless of the HIV status and sexual behavior of men attending the STI clinic of interest for this study. Lifetime smoking and lifetime sex partners were independently associated with any type of oral HPV infection in the multivariate logistic regression model. Future interventions in this type of setting should focus on screening for HPV in the oral cavity as a way of early detection and reduction of malignant changes, HPV-related cancers, and other comorbidities.

\footnotetext{
Abbreviations

HAART: Highly active antiretroviral therapy; HPV: Human papillomavirus; HIV: Human immunodeficiency virus; HIPAA: Health insurance portability and accountability act; HNSCCS: Head and neck squamous cell carcinomas; HR: High-risk; IRB: Institutional review board; Inno LiPA: Innogenetics line price assay; LR: Low-risk; MSM: Male sex partners; MSW: Men who have sex with women; NHANES: National health and nutrition examination survey; PCR: Polymerase chain reaction; PR: Puerto Rico; OPSCCs: Oropharyngeal squamous cell carcinomas; OR: Odds ratio; ORF: Open reading frame; SD: Standard deviation; SEER: Surveillance, epidemiology, and end results; SPF: Small primer fragment; SPSS: Statistical package for the social sciences; STATA: Data analysis and statistical software; STI: Sexually transmitted infection; UPR MSC: University of Puerto Rico-Medical Sciences Campus; US: United States.
}

\section{Competing interests}

The authors declare that they have no competing interests.

\section{Authors' contributions}

VCL conceived of the study, participated in its design and coordination, and helped draft the manuscript. VQA performed the statistical analysis and drafted the manuscript. APO conceived of the study, participated in its design and coordination, and helped draft the manuscript. MS and MMF analyzed the laboratory data, participated in its design and helped draft the manuscript. LMDM, KR, MER, KN participated in data design, study logistics, coordination, and helped draft the manuscript. All authors read and approved the final manuscript.

\section{Acknowledgements}

The project described above was part of the NIH/NIDA AIDS-Science Track Award for Research Transition (ASTART grant: 1 R03 DA031590-01). This study was also partially supported by RCMI grants G12RR003051 (National Center for Research Resources) and G12MD007600 (National Institute on Minority Health and Health Disparities) from the National Institutes of Health. The manuscript's contents are solely the responsibility of the authors and do not necessarily represent the official view of the sponsors $(\mathrm{NIH})$ or the PR Department of Health. Sponsors of this study had no part in the design, data collection, analysis, or interpretation of the findings of this study and did not take part in the writing of or decision to publish this manuscript.

\section{Author details}

${ }^{1}$ Cancer Control and Population Sciences Program, University of Puerto Rico Comprehensive Cancer Center, San Juan, Puerto Rico. ${ }^{2}$ Department of Health Services Administration, Graduate School of Public Health, University of Puerto Rico, San Juan, Puerto Rico. ${ }^{3}$ UPR/MDACC: Partnership for Excellence in Cancer Research Program, School of Medicine, University of Puerto Rico, San Juan, Puerto Rico. ${ }^{4}$ Universidad Autónoma de Guadalajara, Zapopan, Mexico. ${ }^{5}$ Río Piedras Campus, University of Puerto Rico, San Juan, Puerto Rico. 'University of Puerto Rico Comprehensive Cancer Center, San Juan, Puerto Rico. ${ }^{7}$ Department of Pharmaceutical Sciences, School of Pharmacy, University of Puerto Rico, San Juan, Puerto Rico. ${ }^{8}$ Department of Biostatistics and Epidemiology, Graduate School of Public Health, University of Puerto Rico, San Juan, Puerto Rico.

Received: 20 September 2013 Accepted: 9 January 2014 Published: 24 January 2014

\section{References}

1. Suarez TP, Kelly JA, Pinkerton SD, et al: Influence of a partner's HIV serostatus, use of highly active antiretroviral therapy, and viral load on perceptions of sexual risk behavior in a community sample of men who have sex with men. J Acquir Immune Defic Syndr 2001, 28(Suppl 5):471-477.

2. Pagliusi S: Vaccines against Human Papillomavirus: World Health Organization; Retrieved March 25, 2013 from the World Wide Web: http://www.who.int/ vaccines/en/hpvrd.shtml.

3. Gillison ML, Koch WM, Capone RB, et al: Evidence for a causal association between human papillomavirus and a subset of head and neck cancers. J Natl Cancer Inst 2009, 92(Suppl 9):709-720.

4. Chatervedi AK, Engels EA, Pfeiffer RM, et al: Human papillomavirus and rising oropharyngeal cancer incidence in the United States. J Clin Oncol 2008, 29: . Doi: 10.1200/JCO.2011.36.4596.

5. Blomberg M, Nielsen A, Munk, et al: Trends in head and neck cancer incidence in Denmark, 1978-2007: focus on human papillomavirus associated sites. Int J Cancer 2011, 129:733-741.

6. Chatervedi AK, Engels EA, Pfeiffer RM, et al: Human papillomavirus and rising oropharyngeal cancer incidence in the United States. J Clin Oncol 2011, 29: . 10.1200/JCO.2011.36.4596.

7. Auluck A, Hislop G, Bajdik C, et al: Trends in oropharyngeal and oral cavity cancer incidence of human papillomavirus (HPV)-related and HPVunrelated sites in a multicultural population: the British Columbia experience. Cancer 2010, 116:2635-2644.

8. Doobaree IU, Landis SH, Linklater KM, et al: Head and neck cancer in south east England between 1995-1999 and 2000-2004: an estimation of incidence and distribution by site, state and histological type. Oral Oncol 2009, 45:809-814.

9. Braakhuis BJM, Visser O, Leemans R: Oral and oropharyngeal cancer in the Netherlands between 1989 and, increasing incidence, but not in young adults. Oral Oncol 2009, 45(9):e85-e89.

10. Parisi SG, Cruciani M, Scaggiante R, et al: Anal and oral human papillomavirus (HPV) infection in HIV-infected subjects in northern Italy: a longitudinal cohort study among men who have sex with men. BMC Infect Dis 2011, 11:150. 
11. Gillison ML, D' Souza G, Westra W: Distinct risk factor profiles for human papillomavirus type 16-positive and human papillomavirus type 16negative head and neck cancers. JNCI 2008, 100:1-14

12. Bosch FX, Broker TR, Forman D, et al: Comprehensive control of HPV infections and related diseases. Vaccine 2013, 30(7):H1-H31.

13. Kreimer AR, Clifford GM, Boyle P, Franceschi S: Human papillomavirus types in head and neck squamous cell carcinomas worldwide: a systematic review. Cancer Epidemiol Biomarkers Prev 2005, 14(Suppl 2):467-475.

14. Chaturvedi AK, Engels EA, Anderson WF, et al: Incidence trends for human papillomavirus-related and -unrelated oral squamous cell carcinomas in the United States. J Clin Oncol 2008, 26:612-619.

15. Blot WJ, McLaughlin JK, Devesa SS, Fraumeni JF: Cancers of the oral cavity and pharynx. In Cancer Epidemiology and Prevention. Edited by Schottenfeld D, Fraumeni JF Jr. New York: Oxford University Press; 1996:666-680.

16. Edwards BK, Brown ML, Wingo PA, et al: Annual report to the nation on the status of cancer 1975-2002, featuring population-based trends in cancer treatment. J Natl Cancer Inst 2005, 97:1407-1427.

17. Gillison ML, Broutian T, Pickard RK, et al: Prevalence of oral HPV infection in the United States, 2009-2010. JAMA 2012, 307(Suppl 7):693-703.

18. Lu B, Viscidi R, Lee J, et al: Human papillomavirus (HPV) 6,11, 16, and 18 Seroprevalence is associated with sexual practice and age: results from the multinational HPV infection in men study. Canc Epidemiol Biomarkers Prev 2011, 20(Suppl 5):990-1002.

19. Sirera G, Videla S, Piñol M, et al: High prevalence of human papillomavirus infection in the anus, penis and mouth in HIV positive men. Australian Federation of AIDS Organizations 2006, 20(Suppl 8):1201-1204.

20. Kreimer AR, Alberg AJ, Daniel $R$, et al: Oral human papillomavirus infection in adults is associated with sexual behavior and HIV serostatus. JID 2004, 189(4):686-698.

21. Chaturvedi AK: Beyond cervical cancer: burden of other HPV-related cancers among men and women. J Adolesc Helth 2010, 46(Suppl 4):S20-S26.

22. Lowy DR, Schiller JT: Reducing HPV-associated cancer globally. Cancer Prev Res 2012, 5(Suppl 1):18-23.

23. Petti S: Lifestyle risk factors for oral cancer. Oral Oncol 2009, 45(Suppl 4-5):340-350.

24. Hayes RB, Bravo-Otero E, Kleinman DV, et al: Tobacco and alcohol use and oral cancer in Puerto Rico. Cancer Causes Control 1999, 10(Suppl 1):27-33.

25. Day GL, Blot WJ, Austin DF, Bernstein L, et al: Racial differences in risk of oral and pharyngeal cancer: alcohol, tobacco, and other determinants. J Natl Cancer Inst 1993, 85(Suppl 6):465-473.

26. Suárez E, Calo WA, Hernández EY, et al: Age-standardized incidence and mortality rates of oral and pharyngeal cancer in Puerto Rico and among Non-Hispanics whites, Non-Hispanic blacks, and Hispanics in the USA. BMC Cancer 2009, 9:129.

27. Figueroa-Vallés NR, Ortiz-Ortiz KJ, Pérez-Ríos N, Villanueva-Rosa E, TraversoOrtiz M, Torres-Cintrón CR: Suárez-Ramos T (eds): Cancer in Puerto Rico, 2005-2009. San Juan, PR: Puerto Rico Central Cancer Registry; 2012.

28. Alonso I, Felix A, Torné A, et al: Human papillomavirus as a favorable prognostic biomarker in squamous cell carcinomas of the vagina. Gynecol Oncol 2012, 125(Suppl 1):194-199.

29. Szostek S, Klimek M, Zawilinska B, et al: Genotype-specific human papillomavirus detection in cervical smears. Acta Biochim Pol 2008, 55(Suppl 4):687-692

30. Centers for Disease Control and Prevention (CDC): National Health and Nutrition Examination Survey (NHANES): HPV Rinse 2009-2010; http://www. cdc.gov/nchs/data/nhanes/nhanes_09_10/HPV.pdf]

31. Beachler DC, Weber KM, Margolick JB: Risk factors for oral HPV infection among a high prevalence population of HIV-positive and at-risk HIV-negative adults. Cancer Epidemiol Biomarkers Prev 2012, 21(Suppl1):122-133.

32. Goldstone S, Palefsky JM, Giuliano AR, et al: Prevalence of and risk factors for human papillomavirus (HPV) infection among HIV-seronegative men who have sex with men. J Infect Dis 2011, 203(Suppl 1):66-74

33. Kreimer AR, Villa A, Nyitray AG, et al: The epidemiology of oral HPV infection among a multinational sample of healthy men. Cancer Epidemiol Biomarkers Prev 2011, 20(Suppl 1):172-182.

34. Mooij SH, Boot HJ, Speksnijder AG, et al: Oral human papillomavirus infection in HIV-negative and HIV-infected men who have sex with men: The HIV \& HPV in MSM (H2M) study. AIDS 2013.
35. Read TR, Hocking JS, Vodstrcil LA, et al: Oral human papillomavirus in men having sex with men: risk-factors and sampling. PLoS One 2012, 7(Suppl 11):e49324.

36. Parisi SG, Cruciani M, Scaggiante $R$, et al: Anal and oral human papillomavirus (HPV) infection in HIV-infected subjects in northern Italy: a longitudinal cohort study among men who have sex with men. BMC Infect Dis 2011, 11:150-2334-11-150.

37. García-Piñeres AJ, Hildesheim A, Herrero R, et al: Persistent human papillomavirus infection is associated with a generalized decrease in immune responsiveness in older women. Cancer Res 2006 66(Suppl 22):11070-11076

38. Castle PE, Schiffman M, Herrero R, et al: A prospective study of age trends in cervical human papillomavirus acquisition and persistence in Guanacaste. Costa Rica. J Infect Dis 2005, 191(Suppl 11):1808-1816.

39. Proia NK, Paszkiewicz GM, Nacsa MA, et al: Smoking and smokeless tobacco-associated human buccal cell mutations and their association with oral cancer-a review. Cancer Epidemiol Biomarkers Prev 2006 15:1061-1077.

40. Mishra A, Verma P, Marathe N, Srivastava D: Study of the Profile of Patients with STDs Attending an STD Clinic in J.A.H., Gwalior. Indian J Community Med 2008, 33(Suppl 4):263-264.

41. Reddy P, Meyer-Weitz A, Van den Borne B, Kok G: STD-related knowledge, beliefs and attitudes of Xhosa-speaking patients attending STD primary health-care clinics in South Africa. Int J STD AIDS 1999, 10(Suppl 6):392-400.

doi:10.1186/1472-6831-14-7

Cite this article as: Colon-López et al:: Oral HPV infection in a clinicbased sample of Hispanic men. BMC Oral Health 2014 14:7.

\section{Submit your next manuscript to BioMed Central and take full advantage of:}

- Convenient online submission

- Thorough peer review

- No space constraints or color figure charges

- Immediate publication on acceptance

- Inclusion in PubMed, CAS, Scopus and Google Scholar

- Research which is freely available for redistribution 